\title{
Educaplay como recurso didáctico interactivo dirigido a estudiantes de la asignatura Mercadeo
}

\author{
Educaplay as an interactive teaching resource aimed at \\ students of the Marketing subject
}

\author{
José Miguel Salinas Gañango \\ jmsag1494@gmail.com \\ Código ORCID: 0000-0001-8008-6534
}

\author{
Antonella Salvati \\ antonellsalvati1993@gmail.com \\ Código ORCID: 0000-0002-8980-9898
}

Ministerio del Poder Popular para la Educación, Venezuela

Recibido febrero 2020 / Arbitrado marzo 2020 / Publicado mayo 2020

\section{Resumen}

El propósito de la presente investigación fue proponer la herramienta Educaplay como recurso interactivo dirigido al fortalecimiento de los procesos de aprendizaje en los estudiantes de Mercadeo. Las bases teóricas fueron el constructivismo, el aprendizaje significativo, el aprendizaje colaborativo y el conectivismo. La metodología de la investigación se realizó en la modalidad de proyecto factible. La muestra seleccionada fue de tipo estratificado proporcional, en cuanto al instrumento de recolección de la información aplicado fue un cuestionario de 16 preguntas con alternativas de respuestas policotómicas, el cual fue sometido a juicio de expertos y al estudio de confiabilidad a través del alpha de cronbach. En conclusión, se pudo evidenciar que en el proceso de enseñanza de la asignatura Mercadeo no se utilizan las TIC (Técnicas de Información y Comunicación) y no se ha aplicado ninguna herramienta de internet para realizar las actividades, desaprovechando los diferentes beneficios educativos que ofrecen los recursos tecnológicos

\footnotetext{
Abstract

The purpose of this research was to propose the Educaplay tool as an interactive resource aimed at strengthening the learning processes in Marketing students. The theoretical bases were constructivism, meaningful learning, collaborative learning, and connectivism. The research methodology was carried out in the feasible project mode. The selected sample was of a proportional stratified type, in terms of the information collection instrument applied it was a questionnaire of 16 questions with alternatives of polycotomic responses, which was submitted to expert judgment and to the reliability study through the Cronbach's alpha. In conclusion, it was possible to show that in the teaching process of the Marketing subject ICT (Information and Communication Techniques) are not used and no internet tool has been applied to carry out the activities, wasting the different educational benefits offered by the technological resources
}

Palabras clave:

Educaplay; recursos; herramientas tecnológicas; mercadeo 


\section{INTRODUCCIÓN}

L a educación tiene como función formar ciudadanos capaces de enfrentar las transformaciones que a diario se presentan en la sociedad. De este modo se establecen los valores indispensables en pro de una civilización que desea grandes avances. Para lograrlo es necesario una actualización pedagógica en los procesos de enseñanza tradicionales dentro de la universidad, municipio o región, sobre todas las situaciones de aprendizaje, incluida el aula del centro escolar.

Ahora bien, la educación actual persigue la transformación y capacitación profesional de las personas, proporcionándoles herramientas y conocimientos para enfrentar los retos de la vida actual. Desde esta perspectiva se destaca la importancia que tiene la enseñanza de formar profesionales más competentes, es decir, que estén a la altura de los cambios que se producen de manera exponencia a nivel mundial.

Es por esta razón que las tecnologías de información y comunicación desempeñan un papel protagónico en este proceso de transformación de la sociedad. Los avances tecnológicos han contribuido al desarrollo integral de la humanidad, satisfaciendo las necesidades de los seres humanos a través de la historia, permitiéndoles gestionar diversas actividades a través de su simplificación y sistematización.

Actualmente, la sociedad se ha enfrentado a distintos cambios de paradigmas en general. La forma de pensar, de actuar, incluso las formas de trabajar son distintas a como lo eran hace dos generaciones. Todos estos cambios tienen gran influencia en el estilo de vida. Es por esta razón que surge la necesidad de adaptar la educación al nuevo modelo de pensamiento y por supuesto, al uso de las nuevas tecnologías. Es decir, actualizar los centros educativos haciendo de ellos un espacio más flexible, mejor dotados de recursos y fortaleciendo la formación del docente y el estudiante.

En este sentido, los avances tecnológicos exigen a los docentes una mayor preparación, ya que supone la necesidad de incursionar en nuevas competencias, habilidades y dominios técnicos, lo cual implica necesariamente que los profesores participen en planes de formación, actualización y aprendan a emplear las nuevas tecnologías en las actividades académicas, con el objetivo de facilitar y estimular en los estudiantes el placer del aprendizaje. 
De la misma manera, la incorporación de las tecnologías de información y comunicación (TIC) y los recursos tecnológicos en los procesos de enseñanza y aprendizaje, hacen necesario aclarar el modelo pedagógico bajo el cual se desenvuelve el docente, pues es su función principal como uno de los protagonistas de este proceso, es desarrollar actividades orientadas hacia el diagnóstico, la toma de decisiones y la evaluación; tomando en cuenta, que las tecnologías son recursos y como tales, deben insertarse de manera natural en los planes y actividades didácticas, lo que le permita al proceso de enseñanza y aprendizaje encaminarse hacia las nuevas metodologías.

Se puede decir que son muchas las investigaciones realizadas que se vinculan con esta problemática, como por ejemplo el caso de Guanipa (2015), cuyo objetivo general fue proponer estrategias interactivas para el aprendizaje de la asignatura "innovaciones tecnológicas aplicadas a la gerencia educativa" basándose en el aprendizaje colaborativo, cooperativo y significativo. Como conclusión de ese trabajo, se pudo observar que las estrategias interactivas como: la caza de tesoro, webquest, blog, correos conjuntos, cmap tools, mindmanager, educaplay y jclip, constituyen estrategias didácticas donde el estudiante se vuelve más participativo, proactivo, progresa a su propio ritmo y atiende sus diferencias individuales, logrando así, un aprendizaje significativo con mayor rendimiento académico.

El antecedente de investigación descrito, guardó una estrecha relación con el presente estudio, ya que los resultados estuvieron orientados en lograr un aprendizaje significativo a partir de una metodología de enseñanza distinta a la tradicional y mediante el empleo de estrategias innovadoras.

Otra investigación relevante fue la realizada por Pérez (2014) llevada a cabo en la Universidad de Lima. El autor plantea la importancia que tiene Educaplay como plataforma para la comprensión y producción de la materia inglés en alumnos de secundaria. La investigación tuvo como objetivo explicar cómo influye el uso pedagógico de la plataforma Educaplay en el desarrollo de las capacidades de comprensión y producción de textos del idioma inglés. Como resultado se obtuvo que, de dos grupos de estudio donde el primero empleó la herramienta tecnológica Educaplay, se observó que mejoró considerablemente su dominio del tema mientras que, en el segundo grupo que continuó trabajando con métodos tradicionales para la adquisición de nuevos conocimientos disminuyó el nivel 
académico. Por lo tanto, en dicho estudio se considera que la herramienta es eficiente.

En vista de lo dicho anterior mente, se puede considerar esta herramienta como una estrategia de enseñanza que permite la creación de actividades educativas multimedia que se pueden emplear en el aula con los estudiantes. Además, se pueden crear colecciones de actividades y grupos tanto con alumnos como con otros compañeros y docentes. Dentro de las actividades que permite crear el recurso digital Educaplay se encuentran las siguientes: completación, test, presentaciones, relación, evaluaciones, entre otras. Dentro de este trabajo de investigación se considera a Educaplay como el recurso educativo que permitirá innovar y actualizar la manera de aprender en la asignatura de Mercadeo.

Cabe destacar que en Venezuela se cuentan con fundamentos legales que respaldan esta propuesta. En primer lugar, se tiene la Constitución de la República Bolivariana de Venezuela (1999) en el artículo 108 alusivo a la garantía que debe brindar el Estado y el papel que tienen los medios en la formación ciudadana:

Los medios de comunicación social, públicos y privados, deben contribuir a la formación ciudadana. El Estado garantizará servicios públicos de radio, televisión y redes de bibliotecas y de informática, con el fin de permitir el acceso universal a la información. Los centros educativos deben incorporar el conocimiento y aplicación de las nuevas tecnologías, de sus innovaciones, según los requisitos que establezca la ley. (p.37)

De este modo, el estado venezolano es uno de los responsables de garantizar el acceso a la comunicación y a las nuevas tecnologías. En el ámbito educativo, a través de este artículo, se le da un carácter constitucional al uso de Educaplay como recurso interactivo para el fortalecimiento del aprendizaje. Aunado a esto se tiene la Ley Orgánica de Educación (2009) en su artículo 9 donde establece:

En los subsistemas del Sistema Educativo se incorporan unidades de formación para contribuir con el conocimiento, comprensión, uso y análisis crítico de contenidos de los medios de comunicación social. Asimismo, la ley y los reglamentos regularán la propaganda en defensa de la salud mental y física de la población. (p.14) 
Es por tanto que, la práctica docente requiere de profesionales de la educación que estén preparados para el uso de las nuevas tecnologías y que además de tener las herramientas necesarias a su alcance, tengan el interés por aplicarlas en su labor pedagógica y así garantizar la asimilación de los conocimientos a sus educandos de forma trascendente. Un docente que domine estas metodologías es garantía de que el sistema educativo se pueda actualizar y mejorar. En concordancia con la Unesco (2010) "Las nuevas generaciones son ya nativas digitales y muestran inéditas formas de comunicarse, de entretenerse y de socializar. Por contraste, las escuelas y sus prácticas siguen ancladas en el siglo XIX."(párr. 5)

Sin embargo, la educación actual en Venezuela se encuentra estancada en esta realidad. No solamente por el acceso limitado de algunos sectores a los recursos tecnológicos sino por el empleo de metodologías poco significativas del sistema tradicional. Una forma de contribuir a la solución de esta problemática es rompiendo los paradigmas y actualizando el viejo esquema en el que aún se encuentran algunos sectores de la educación venezolana. De este modo, crear un nuevo esquema que permita desarrollar un aprendizaje significativo, cooperativo y participativo.

Otro aspecto a corregir para mejorar el sistema educativo actual es la resistencia al cambio. Esta juega un papel antagónico para el avance que pretende la sociedad, ya que no permite la innovación en la educación ni la incorporación de las herramientas que se requieren en esta nueva era de la información y la comunicación. Como respaldo a esta última idea, es relevante citar el Decreto 825 de la Gaceta Oficial Nº 36955 (2000) en su artículo 11 donde expresa lo siguiente:

El Estado, a través del Ministerio de Ciencia y Tecnología promoverá activamente el desarrollo del material académico, científico y cultural para lograr un acceso adecuado y uso efectivo de Internet, a los fines de establecer un ámbito para la investigación y el desarrollo del conocimiento en el sector de las tecnologías de la información. (p.2)

En tal sentido el Estado, a través del Ministerio de Ciencia considera que generar políticas adecuadas para el uso y fomento de las TIC, es de suma importancia para el desarrollo integral de los estudiantes de cualquier área del conocimiento. Eso incluye la 
educación superior, la cual no escapa al proceso de cambio al que debe estar sujeta la educación venezolana, aprovechando las herramientas y nuevas estrategias interactivas que le permitan al estudiantado apropiarse del conocimiento y utilizarlo oportunamente.

Como respuesta a la problemática expuesta anteriormente, la presente investigación se plantea la siguiente interrogante: ¿De qué manera Educaplay podría fortalecer los procesos de aprendizaje en los estudiantes la asignatura Mercadeo de la Facultad de Educación de la Universidad de Carabobo? A razón de dicha interrogante, se planteó como objetivo general proponer la herramienta Educaplay como recurso interactivo dirigido al fortalecimiento de los procesos de aprendizaje en los estudiantes de la asignatura Mercadeo.

Una vez planteado el objetivo general de la investigación, fue necesario, en primera instancia, diagnosticar la necesidad de Educaplay como recurso interactivo para el fortalecimiento de los procesos de aprendizaje en los estudiantes de mercadeo. Seguidamente, determinar la factibilidad de la propuesta. Finalmente, diseñar estrategias mediante Educaplay como recurso interactivo para el fortalecimiento de los procesos de aprendizaje en los estudiantes de mercadeo.

En concordancia con lo anteriormente dicho, el desarrollo de esta investigación surgió como respuesta a la necesidad de incorporar nuevas estrategias y recursos de enseñanza aprendizaje, enmarcados en el uso y aprovechamiento de las tecnologías de información que mejoren y optimicen la educación que reciben los estudiantes de la asignatura de Mercadeo en la institución antes mencionada.

Debido al contexto de aplicación de esta investigación se considera relevante explicar qué es el Mercadeo. De este modo, se le puede definir como todo lo que se haga para promover una actividad. Esto abarca desde el momento en que se concibe la idea, hasta la etapa en que los clientes comienzan a adquirir el producto o servicio en una base regular. En este sentido, el mercadeo implica la acción de ponerle nombre a una empresa o producto, seleccionar el producto, la determinación del lugar donde se venderá el dicho producto o servicio, el color, la forma, tamaño, el empaque, la localización del negocio, la publicidad, las relaciones públicas, el tipo de venta que se hará, el entrenamiento de ventas, la presentación de ventas, la solución de problemas, el plan estratégico de crecimiento, y el seguimiento. 
Aunado a esto, en el Mercadeo una de las funciones primordiales es crear y desarrollar el ingenio, la inventiva y la creatividad de cada estudiante. Es por esta razón que el Mercadeo está incluido dentro del pensum de la Mención de Educación para el Trabajo en la institución ya mencionada, pues es fundamental en el perfil del egresado de esta mención conocer todo lo referente a esta área.

Con respecto a esta idea, es relevante decir que el empleo de Educaplay como recurso interactivo en la asignatura de Mercadeo tuvo un impacto muy positivo porque le brindó mayor calidad al proceso educativo de la población de estudio la cual, lamentablemente se encontraba atascada en un sistema de enseñanza poco significativo y descontextualizado que, a pesar de estar en un contexto globalizado en plena era de la información y los recursos digitales, no aprovechaba las herramientas tecnológicas a su disposición.

Se puede decir entonces que el modelo educativo actual no corresponde con la realidad de una sociedad que se encuentra inmersa en las nuevas tecnologías de información y comunicación. Pareciera más bien que la educación se quedó detenida en los modelos ortodoxos. Es por esta razón que surge la necesidad de un cambio de paradigma que incluya a los recursos tecnológicos como parte del proceso educativo y se garantice el aprendizaje. Al respecto, Siemens (2004) define el aprendizaje como:

Proceso que ocurre al interior de ambientes difusos de elementos centrales cambiantes, que no están por completo bajo control del individuo. El aprendizaje puede residir fuera de nosotros, está enfocado en conectar conjuntos de información especializada, y las conexiones que nos permiten aprender más tienen mayor importancia que nuestro estado actual de conocimiento. (p.18)

Sustentado en lo anteriormente descrito, el uso de las actividades de Educaplay como recurso interactivo permitirá complementar los contenidos de la asignatura Mercadeo que se imparten mediante estrategias obsoletas y además, como este recurso ofrece actividades creativas e innovadoras, se ajusta a los ritmos de aprendizaje de la actualidad que crecen y se diversifican de manera exponencial.

Tomando en cuenta lo dicho por el autor citado, quien planteó que la educación debe ser transcendental, queda en evidencia que se deben romper los viejos paradigmas y salir de los métodos tradicionales para que así se propicie el aprendizaje significativo. En 
consonancia con lo planteado por Ausubel (1973) en su teoría, "el Aprendizaje esta producido en un contexto educativo, es decir en el marco de una situación de interiorización o asimilación a través de la instrucción" (p. 209).

\section{MÉTODO}

continuación, se presenta la metodología que se empleó en el $\triangle$ desarrollo de la investigación. En esta parte se incluye el tipo 1 de investigación, las técnicas y procedimientos que se utilizaron para llevar a cabo el estudio. En pocas palabras, el "cómo" se realizó la ruta metodológica para responder al problema planteado.

La presente investigación se realizó bajo el paradigma cuantitativo. La tipología de esta investigación es de un proyecto factible. Al respecto, Palella y Martins (2010) señalan que: “Consiste en la investigación, elaboración y desarrollo de una propuesta de un modelo operativo viable para solucionar problemas, requerimientos, o necesidades, de organizaciones o grupos sociales; puede referirse a la formulación de políticas, programas tecnologías y métodos o procesos". (p. 116) Por otra parte, la elaboración del proyecto factible está destinada a atender necesidades específicas a partir de un diagnóstico. Según UPEL (2012):

... consiste en la investigación, elaboración y desarrollo de un modelo operativo viable para solucionar problemas, requerimientos necesidades de organizaciones o grupos sociales que pueden referirse a la formulación de políticas, programas, tecnologías, métodos, o procesos. El proyecto debe tener el apoyo de una investigación de tipo documental, y de campo, o un diseño que incluya ambas modalidades. (p.21)

En este sentido la investigación tiene como punto de partida el diagnóstico sobre la aplicación de herramientas interactivas como Educaplay en el proceso educativo de la asignatura Mercadeo. La investigación finaliza con el diseño de herramientas pedagógicas para la incorporación de Educaplay como estrategia de enseñanza en la asignatura de Mercadeo.

Así mismo, para el desarrollo de esta investigación se procedió a trabajar en fases: en primer lugar, se llevó a cabo la Fase I 
denominada diagnóstico de las necesidades; allí se aplicó el instrumento a una muestra a fin de detectar la necesidad de Educaplay como recurso interactivo para el fortalecimiento de los procesos de aprendizaje en los estudiantes de mercadeo.

Seguidamente se realizó la Fase II, denominado es estudio de factibilidad, donde se estudiaron aspectos como el ámbito económico, técnico e institucional y se detectó que la investigación era factible ya que la Facultad de Ciencias de la Educación de la Universidad de Carabobo cuenta con instalaciones que facilitan el desenvolvimiento para la ejecución y evaluación del proyecto, específicamente laboratorios de computación, donde se pudieron realizar las actividades pautadas para el desarrollo académico de los estudiantes de la asignatura. Finalmente se procedió con la Fase III denominada diseño de la propuesta.

En continuidad con la metodología de esta investigación, la misma se apoyó en un diseño de campo, ya que el objeto de estudio surge de una realidad y la información requerida se obtiene de ella. De este modo, la investigación de campo es un método directo para obtener información confiable que permita conocer la situación real del problema e imaginarse las propuestas para solucionarlo. En este sentido, Arias (2006) afirma que:

La investigación de campo, es aquella que consiste en la recolección de datos directamente de los sujetos investigados, o de la realidad donde ocurren los hechos (datos primarios), sin manipular ni controlar variable alguna, es decir, el investigador obtiene la información, pero no altera las condiciones existentes. De allí su carácter de no experimental (...). (p. 31)

En cuanto a la población de estudio, es un conjunto de individuos de la misma clase. Según Hernández, Fernández y Baptista (2010) la población se define como "el conjunto de todos los casos que concuerdan con determinadas especificaciones" (p. 174). En este sentido, la población objeto de estudio de esta investigación estará integrada por la totalidad de 65 estudiantes del sexto semestre de la mención de Educación para el Trabajo sub área Comercial cursantes de la asignatura Mercadeo. De los mismos, para fines de este estudio se estableció una muestra de tipo estratificado proporcional. Por tanto, en el estudio participaron 27 estudiantes cursantes de la asignatura inscritos en el periodo 1/2019. Según Arias (2006): 
La muestra es un subconjunto representativo y finito que se extrae de la población accesible, una muestra representativa es aquella que por su tamaño y características similares a las del conjunto, permiten hacer inferencias o generalizar los resultados al resto de la población con un margen de error conocido. (p. 83)

Cabe destacar que para efectos de la presente investigación también se aplicó una encuesta, que según lo señalado por Sierra (2005) consiste en "la obtención de datos de interés sociológico mediante la interrogación a los miembros de un determinado grupo social (...) consiste en la observación no directa de los hechos, sino a través de las manifestaciones realizadas por los propios interesados" (p. 305). En este sentido, la encuesta representó la fuente idónea para que los sujetos que conforman la muestra, a través de un instrumento de fácil llenado y de lenguaje simple, expresaran sus impresiones sobre la aplicación de herramientas interactivas como Educaplay en las estrategias de enseñanza aprendizaje de la asignatura Mercadeo.

Dicha encuesta se aplicó a los participantes que conforman la muestra, mediante un cuestionario. Este instrumento es un formato donde se encuentran anotadas una serie de preguntas para que el entrevistado las responda. Al respecto Arias (ob. cit.) lo define como "un formato que contiene una serie de preguntas en función de la información que se desea obtener, y que se responde por escrito" (p. 54). La validez del mismo se refiere "al grado en que un instrumento de medición mide realmente la(s) variable(s) que se busca medir" (Hernández, Fernández y Baptista, 2010, p. 201). Esta validez puede ser de tres tipos: validez de contenido, de constructo y a través del juicio de expertos.

En el caso particular de la investigación se recurrió a la validez de contenido. Con respecto a este tipo de validez, "se refiere al grado en que un instrumento refleja un dominio específico de contenido de lo que se mide" (Hernández, Fernández y Baptista, 2010, p. 201) Dicha validez fue realizada a través de la comparación del cuadro de operacionalización de variables con el instrumento diseñado.

De igual manera, se emplea la técnica de validación de juicio de expertos, la cual: "se refiere al grado en que aparentemente un instrumento de medición mide la variable en cuestión, de acuerdo con expertos en el tema" (Hernández, Fernández y Baptista, 2010, 
p. 201). Este tipo de validación consiste en entregarle a tres expertos, un modelo del instrumento junto con los objetivos de la investigación y la operacionalización de las variables a fin que éstos revisen el contenido, redacción y pertinencia de los ítems en el instrumento. De esta manera, el investigador realiza las correcciones pertinentes previas a su aplicación. Para gestionar este tipo de validez, se decidió consultar a tres expertos a través de una petición formal. Luego se efectuaron las correcciones necesarias para su aplicación a la muestra piloto.

En cuanto al cálculo de la confiabilidad, Hernández, Fernández y Baptista (2010) indican que es el "grado en que un instrumento produce resultados consistentes y coherentes" (p. 200). Para realizar el cálculo de la confiabilidad de un instrumento de recolección de datos con características tipo Likert, en el cual no existen respuestas correctas, se procedió a emplear el coeficiente Alfa de Cronbach donde se contrastan la variabilidad de respuestas de todos los ítems por separado y por persona.

A su vez, un instrumento se considera aceptable cuando su coeficiente de confiabilidad es igual o mayor a 0,61 . No obstante, no existe regla fija para todos los casos, esto depende del tipo de instrumento en estudio, de su propósito y del tipo de confiabilidad que se trate. Se sugiere que cuando se usen escalas de actitud, el coeficiente de confiabilidad nunca debe estar por debajo de 0,81 para ser aceptable. En este sentido, el coeficiente de confiabilidad del cuestionario aplicado a los estudiantes de mercadeo es alto, lo cual significa que al aplicar nuevamente el instrumento en condiciones similares existe una "alta" probabilidad de obtener resultados semejantes.

Finalmente, tanto las tablas como los análisis fueron realizados según las diferentes dimensiones e indicadores previamente establecidos para la construcción del instrumento; por ende, los ítems que conformen dicha dimensión se agruparon de acuerdo a las opciones de respuesta, realizando así una interpretación del mismo.

\section{RESULTADOS Y DISCUSIÓN}

$\mathrm{E}$ sta investigación tuvo como objetivo proponer la herramienta Educaplay como recurso interactivo para el fortalecimiento de los procesos de aprendizaje en los estudiantes de mercadeo; respondiendo a la modalidad de Proyecto Factible; para el 
análisis se diseñaron tablas y gráficos estadísticos de acuerdo a las dimensiones e indicadores. El análisis muestra la frecuencia con que los sujetos se ubican en las alternativas de respuesta del cuestionario aplicado: Siempre, Casi siempre, Casi nunca y Nunca, presentándose los respectivos porcentajes.

A continuación, se presentan los resultados obtenidos en los siete primeros ítems durante la aplicación del cuestionario a los estudiantes de la asignatura Mercadeo de la mención Educación para el Trabajo sub área Comercial de la Facultad de Educación de la Universidad de Carabobo perteneciente a la muestra del estudio. En los gráficos se colocaron los datos suministrados por los estudiantes de la asignatura como respuesta a los ítems del cuestionario aplicado.

Asimismo, se realizó la interpretación de los resultados obtenidos, destacando los porcentajes más significativos de las respuestas dadas por los encuestados y explicando el significado de la información en atención a los propósitos del estudio realizado. Por último, se relaciona la información con el basamento teórico que sirvió de soporte a la investigación, para posteriormente reflejar las conclusiones de la presente investigación.

En el caso del ítem 1 que se puede apreciar en la figura 1, estuvo dirigido a determinar el acceso a internet en el aula que posee el estudiante. Se obtuvo que un 33 por ciento de los encuestados consideraron que Nunca tienen acceso a internet en el aula, el 26 por ciento Casi nunca, el 19 por ciento Casi siempre y el 22 por ciento Siempre. Esto permitió afirmar que una porción considerable de los estudiantes, no poseen internet en el aula.

Por otra parte, en el indicador e-actividades del ítem 2 que también se puede apreciar en la figura 1, estuvo dirigido a determinar cómo se refuerzan los contenidos a través de actividades en línea. Se obtuvo que un 63 por ciento de los encuestados consideraron que Nunca se refuerzan contenidos a través de actividades en línea, 37 por ciento Casi nunca. Esto permite aseverar que la mayoría de la muestra seleccionada Nunca refuerza contenidos a través de actividades en línea en la asignatura Mercadeo.

De igual forma en el Ítem 3, dirigido a determinar si el estudiante ha realizado evaluaciones a través de internet, se obtuvo que un 74 por ciento de los encuestados consideraron que Nunca han realizado evaluaciones a través de internet, 26 Casi nunca. Esto deja en evidencia que la mayoría de los estudiantes encuestados Nunca han 
realizado evaluaciones a través de internet en la asignatura Mercadeo. Estos resultados se pueden apreciar con más detalle en la figura 1.

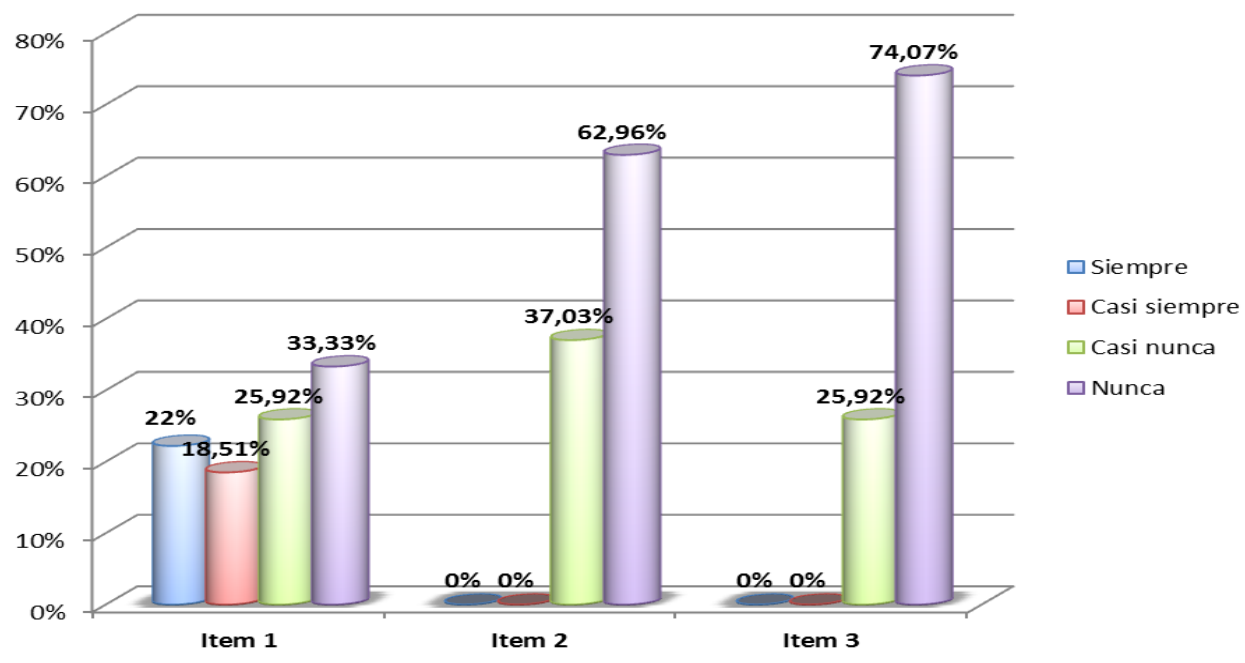

Figura 1. Dimensión Educaplay. (Fuente: Salinas y Salvati, 2020)

Según lo anteriormente descrito en los ítems 1,2 y 3 se puede apreciar que la mayoría de los estudiantes encuestados Nunca: han tenido acceso internet en el aula, no realizan actividades de mercadeo en internet ni evaluaciones de mercadeo a través de la misma. Estos resultados motivan aún más a implementar Educaplay como recurso interactivo para el fortalecimiento de los conocimientos y aprendizajes de los estudiantes de mercadeo.

En a la dimensión Multimedia que se puede apreciar con mayor claridad en la figura 2, se midió el indicador audio a través del ítem 4 dirigido a determinar cómo el estudiante en clases de mercadeo, realiza actividades en las cuales utiliza el Audio como estrategia interactiva. Se obtuvo que un 74 por ciento de los encuestados consideraron que Casi nunca se utiliza audio como estrategia en el aula, 15 por ciento Nunca, 11 por ciento Casi siempre.

En este mismo orden de ideas, en el ítem 5 de la dimensión ya descrita, se mide el indicador Video con el fin de determinar con qué frecuencia el estudiante considera que, en clases de mercadeo, realiza actividades en las cuales se utilice el Video como estrategia interactiva. Se obtuvo que un 52 por ciento respondió Casi nunca y un 48 por ciento Nunca. 
Más adelante, en el indicador Animación correspondiente al ítem 6, dirigido a determinar: con qué frecuencia el estudiante en clases de mercadeo realiza actividades en las cuales utiliza la Animación como estrategia interactiva, se obtuvo que un 59 por ciento de los encuestados consideren que Siempre usan animaciones en la asignatura, 30 por ciento Casi siempre.

De igual forma, el ítem 7 con el indicador Texto, estuvo dirigido a determinar: con qué frecuencia el estudiante considera que en clases de mercadeo realiza actividades en las cuales utilice el texto como estrategia interactiva. Se obtuvo que un 74 por ciento de las respuestas apuntaron a Siempre mientras que el 26 por ciento fueron casi siempre se utiliza el texto como estrategia para la asignatura mercadeo. Todos estos resultados se pueden apreciar a continuación en la figura 2 .

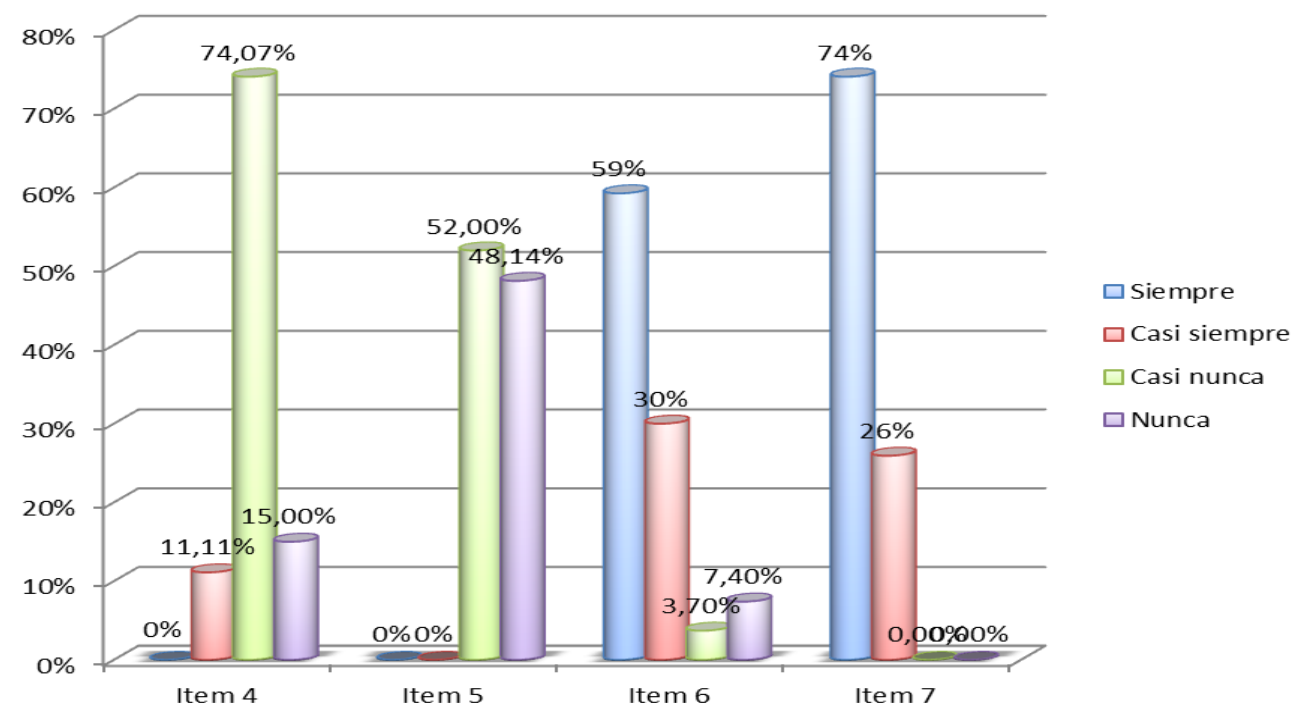

Figura 2. Dimensión Multimedia. (Fuente: Salinas y Salvati, 2020)

\section{CONCLUSIONES}

$\mathrm{D}$ e acuerdo a los resultados obtenidos en el instrumento aplicado a los estudiantes de mercadeo del sexto semestre de la Facultad de Educación de la Universidad de Carabobo, se pudo evidenciar que en lo relacionado al objetivo de proponer la herramienta Educaplay como recurso interactivo para el fortalecimiento, optimización y mejora de los procesos de aprendizaje en los estudiantes de mercadeo, es factible y necesario ya que el 70 
por ciento de los estudiantes nunca utilizan el recurso Educaplay y cualquier otro recurso tecnológico como medio de enseñanza.

Por esta razón, es indispensable la formación de los estudiantes de la asignatura en el manejo adecuado de las Tecnologías de la Información y la Comunicación (TIC). Dicha formación ayudará a garantizar la inclusión de esta valiosa herramienta en el sistema educativo y de esta manera, se colabore a mejorar el desempeño del estudiante y a su vez el del docente.

En atención al objetivo dos, el cual buscó determinar la factibilidad de incorporación de Educaplay como recurso interactivo para el fortalecimiento de los procesos de aprendizaje en los estudiantes de mercadeo se concluyó que la Facultad de Educación, cuenta con laboratorios de computación y también con los materiales necesarios para el desenvolvimiento, ejecución y evaluación del programa, lo que se traduce en un gran beneficio para el desarrollo académico de la mención.

Por otra parte, se puede evidenciar que el 60 por ciento de los estudiantes están dispuestos, motivados y también, les gustaría que en la mención de Educación para el Trabajo se realicen talleres de formación dirigidos a los docentes, donde se les capacite en el desarrollo de herramientas digitales y de esta manera, mantenerse actualizados y diestros en la ejecución del proceso de enseñanza. Esto es garantiza el desarrollo integral de los estudiantes y sus múltiples capacidades.

Como otro aspecto favorable en estas conclusiones, se puede decir que implementación del recurso interactivo Educaplay beneficia en gran medida al docente, ya que 70 por ciento de ellos no utilizan los recursos tecnológicos, y por otro lado existe un 60 por ciento de disposición por parte de los estudiantes a aplicarlo. Cabe destacar que los mismos se sienten motivados en emplear la tecnología e incluirlas como método en la praxis diaria, lo que conlleva a enriquecer el desarrollo profesional de los participantes del hecho educativo.

Es importante tomar en cuenta que una de las limitaciones que tiene el departamento de administración y planeamiento educativo de la facultad de educación es la de no realizar prácticas relacionadas a las TIC dentro de la asignatura Mercadeo. Esto trae como consecuencia que el proceso educativo esté arraigado a una metodología tradicional para impartir las clases y realizar las evaluaciones.

En este mismo orden de ideas, y como respuesta a la limitante descrita, la presente investigación propone el empleo de herramientas 
tecnológicas más contemporáneas en el proceso educativo. Específicamente se recomienda la herramienta interactiva Educaplay como estrategia pedagógica en de trabajo de la asignatura. De este modo, se puede actualizar la modalidad de ejecutar las evaluaciones y los contenidos de dicha asignatura.

Atendiendo a los términos referidos, la estructura de la propuesta incluyó los siguientes aspectos: Fase I: Las TIC en el acto didáctico; Fase II: Elaboración y construcción de actividades para la Asignatura Mercadeo. Fase III: Construcción de evaluaciones mediante la herramienta interactiva Educaplay. Todo esto se realizó con la finalidad de motivar a los estudiantes y profesores a conocer la herramienta interactiva Educaplay, capacitar a los docentes de la asignatura mercadeo acerca de la herramienta, elaborar contenidos a desarrollar en la plataforma de Educaplay y realizar construcciones de actividades lúdicas y de interacción dentro de la herramienta interactiva.

En concordancia con lo anteriormente descrito, el diseño de la propuesta pretendió ser útil para la ejecución de planes y programas educativos ya que, coinciden con la necesidad que tienen los docentes de desarrollar habilidades y destrezas dentro de la práctica pedagógica. En el mismo orden de ideas, para el desarrollo de esta investigación respondió a la necesidad de incorporar nuevos recursos que potencien el aprendizaje en los estudiantes de la asignatura Mercadeo y, que estén enmarcados en el uso de las tecnologías de información.

En cuanto a la factibilidad de la propuesta, una vez que se evidenció la problemática existente, se pudo determinar la factibilidad de proponer estrategias interactivas para el aprendizaje de la asignatura, por lo tanto, el estudio del proyecto permitió conocer si es posible o no la propuesta planteada, además de los beneficios, los recursos técnicos y humanos, el costo y financiamiento para el desarrollo y evaluación de dicha propuesta.

Como conclusión final es posible asegurar que la herramienta Educaplay colaboró a mejorar el perfil académico del estudiante, siendo el Mercadeo una asignatura con tendencia al emprendimiento social y profesional, dicha asignatura debe estar al día con las tendencias y comportamiento de la sociedad. Por esta razón resulta tan beneficioso para los participantes este tipo de propuestas pedagógicas. 


\section{REFERENCIAS}

Arias, F. (2006). El Proyecto de Investigación. Guía para su elaboración. ( $3^{\mathrm{a}}$ ed.) Caracas: Editorial Episteme

Ausubel, D. P. (1973). La educación y la estructura del conocimiento. Buenos Aires: El Ateneo

Constitución de la República Bolivariana de Venezuela (1999) Gaceta Oficial de la República Bolivariana de Venezuela, (Extraordinario), diciembre 30, 1999

Gaceta Oficial de la República de Venezuela No. 36.955 22. (2000) Decreto 825

Guanipa, L. (2015). Estrategias interactivas para el aprendizaje de la asignatura innovaciones tecnológicas aplicadas a la gerencia educativa en la FaCE de la UC. Universidad de Carabobo

Hernández, R., Fernández, C. y Baptista, P. (2010). Metodología de la investigación. (5a ed.).México: McGraw Hill

Hurtado, J. (2010). Metodología de la Investigación. (4 ${ }^{\mathrm{a}}$ ed.) Venezuela: Quirón

Ley Orgánica de Educación (2009) Gaceta Oficial de la República de Venezuela, 5.929. (Extraordinario)

Palella, S. y Martins F. (2010). Metodología de la Investigación cuantitativa. (2 ${ }^{\mathrm{a}}$ ed.) Venezuela: Editorial FEDUPEL

Pérez, N. (2014). Influencia del uso de la plataforma Educaplay en el desarrollo de las capacidades de comprensión y producción de textos en el área de Inglés en alumnos de 1er año de secundaria de una institución educativa de Lima. (Tesis de Maestría). Recuperado de http://tesis.pucp.edu.pe/repositorio/handle/20.500. $12404 / 5589$

Siemens, G. (2004). Conectivismo: Una teoría de aprendizaje para la era digital. Recuperado de https://pdfs.semanticscholar.org/05f1/ adee187323d66

beab226058b23a7416c3517.pdf

Sierra, R. (2005) Técnicas de investigación social. Teorías y ejercicios. (14 ${ }^{\mathrm{a}}$ ed.), Madrid, España: Paraninfo

Unesco. (2010). Las Tecnologías de la Información y la Comunicación en la Formación Docente. Recuperado de http://www.unesco.cl/ medios/biblioteca/documentos/formacion docente tecnologías información comunicación.pd̄f

Universidad Pedagógica Experimental Libertador, UPEL. (2012). Manual de trabajos de grado, de especialización y maestría y tesis doctorales. Caracas: FEDEUPEL 